\title{
Experimental study of flow around two grooved cylinders arranged in tandem
}

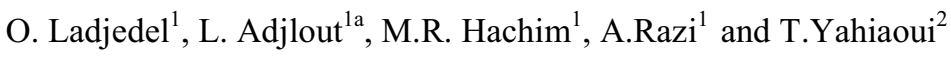 \\ ${ }^{1}$ laboratoire d'aérohydrodynamique naval Département de Génie Maritime Mechanical Engineering Faculty, USTO Oran, \\ Oran 31000 Algeria \\ ${ }^{2}$ Laboratoire d'aéronautique et systèmes propulsive Département de Génie Mécanique Mechanical Engineering Faculty, \\ USTO Oran, Oran 31000 Algeria
}

\begin{abstract}
In the present paper, the influence of longitudinal grooves placed on tubes is investigated for different configurations in tandem for three Reynolds number. Each cylinder has two grooves on the external surface at $90^{\circ}$ and $270^{\circ}$ degrees. The experiments are carried out using a subsonic wind tunnel. The pressure distributions along the tubes are determined for a variation of the azimuthal angle from 0 to 360 degrees. The drag and lift forces are measured using the TE 44 balance. The results show that the upstream cylinder pressure is greater than for second one. There is a difference in the pressure coefficient as well as the pressure drag for configuration smooth-smooth and grooved-grooved.
\end{abstract}

\section{Introduction}

Most structures on land and in the ocean are in multiple forms such as groups of chimney stacks, tubes in heat exchangers, over head power-line bundles, bridge piers, stays, masts, chemical reaction towers, off-shore platforms, adjacent skyscrapers, etc and were confronted by a fluid flow. Fluid flows reduce the life of the respective installations and must therefore be taken into account in the design of the structure. Therefore it was important to understand the interaction of multiple structures in a flow. An elementary shape of a structure or a component of a structure is a circular cross-section, and a tandem arrangement of two circular cylinders is a basic example of an array of multiple structures.

Flow around two tandem circular rigid cylinders has been investigated from the subcritical Re to the super critical (e.g., Okajima, [1]). In general this flow may be classified as three regimes based on L/D (Zdravkovich [2]): (1)extended-body regime, where $\mathrm{L} / \mathrm{D}$ is from 0 to 0.5 and the free shear layers separated from the upstream cylinder over shoot the downstream; (2) reattachment regime, where $\mathrm{L} / \mathrm{D}$ is between 0.5 and 3 , and the shear layers reattach on the downstream cylinder; and (3)co-shedding regime, where $\mathrm{L} / \mathrm{D}>3$ and the shear layers roll up alternately, forming a vortex street in the gap between as well as behind the cylinders. Arie et al. [3] pointed out that fluctuation in drag force acting on both cylinders is weakly dependent on spacing. On the other hand, Igarashi [4] reported that the fluctuation in pressure associated with fluctuation in lift and drag forces acting on a downstream cylinder is strongly dependent on spacing between the cylinders. Moreover, some discrepant points among various studies can be found in [4]. Alam et al. [5] further divided the reattachment regime into two: regime of alternating reattachment of the shear layers $(\mathrm{L} / \mathrm{D}<2)$ and regime of steady reattachment of the shear layers $(\mathrm{L} / \mathrm{D}=2-3)$. The regime of alternating reattachment corresponds to a higher fluctuating lift force coefficient $\mathrm{C}_{\mathrm{Lf}}$ specially on the downstream cylinder and the regime of steady reattachment corresponds to a lower $\mathrm{C}_{\mathrm{Lf}}$ on both cylinders. It should be noted that the classification of the flow regimes for rigid cylinders is dependent on Reynolds number (Re) Xu and Zhou [6]. Naturally, the $\mathrm{L} / \mathrm{D}$ ranges of the five regimes observed for flexible cylinders may differ at a very high Re. Alam et al. [7] performed an experiment to suppress fluid forces on two circular cylinders in tandem arrangement by using two tripping rods on each of the cylinders. They were able to suppress time-averaged drag, fluctuating drag and fluctuating lift of the upstream cylinder by $70 \%, 65 \%$ and $89 \%$, respectively, for any spacing between the cylinders, and also find significant reduction in fluid forces on the downstream cylinder. Many investigations of tandem configurations by Brika and Laneville [8], Laneville and Brika [9], Hover and Triantafyllou [10] and Assi et al. [11] showed substantial complexity in fluid dynamics as the spacing between the cylinders is varied, including a combination of vortex induced vibration and gallopinglike oscillations. When the upstream cylinder is fixed and the downstream cylinder is free to oscillate, both vortex resonance and the occurrence of wake-galloping instability are equally possible and can occur separately or combined depending on the separation distance

\footnotetext{
${ }^{a}$ Corresponding author: aldjloutl@yahoo.fr
} 
according to Bokaian and Geoola [12]. Ladjedel et al [13] found that for a Reynolds number $\mathrm{Re}=8.6 \times 10^{4}$ the cylinder fitted with two longitudinal grooves reduces the drag force about $17.51 \%$. The purpose of the present work is to investigate experimentally the influence and the interest of using grooves on flow around two cylinders in tandem configuration.

\section{Experimental facilities}

The experiments are carried out in closed circuit wind tunnel. The test section is of square cross-section $0.46 \mathrm{~m} \times 0.46 \mathrm{~m}$ and $1.2 \mathrm{~m}$ long. The closed circuit wind tunnel is of conventional design in which the velocity could reach $60 \mathrm{~m} / \mathrm{s}$ and the turbulence intensity is less than $1 \%$.

The test section is composed of two rigid PVC tubes with an identical external diameter of $40 \mathrm{~mm}$ and $460 \mathrm{~mm}$ length. Cylinders are tandem arrangement. As shown in figure 1 .

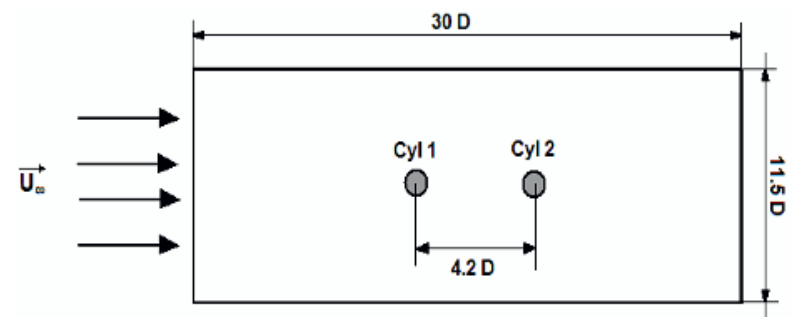

Fig.1. Experimental set up.

The grooved cylinder is equipped with 22 pressure tapings on its circumference and one pressure taping for the smooth cylinder where the latter will be turned from $0^{\circ}$ to $360^{\circ}$ with an interval of $10^{\circ}$. The diameter of the tapings is $0.5 \mathrm{~mm}$ and the location of the tapings for the both cylinders is at mid-span. The shape and dimensions of the groove are shown in Figure (2). The pressure measurements are carried out for the upstream and the downstream cylinder for an azimuthal angle from $0^{\circ}$ to $360^{\circ}$. For details of the wind tunnel and the experimental setup (see Ladjedel et al. [13]).

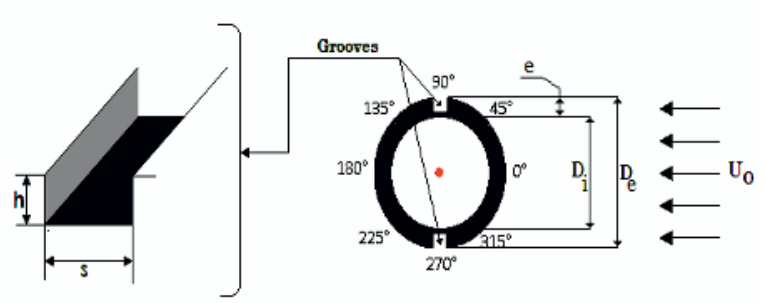

Fig.2. Shape and dimension of the grooves.

\section{Results and discussion}

Two cases are investigated, the first one is SmoothSmooth cylinders and the second case is groovedgrooved cylinders. The experimental study is divided into two main parts:

a) Measurement of pressure around the cylinder for three Reynolds number: $\operatorname{Re}_{1}=2 \times 10^{4}, \mathrm{Re}_{2}=5 \times 10^{4}$ and $\mathrm{Re}_{3}=7 \times 10^{4}$ (based on diameter and Free stream velocity); b) Measurement of drag and lift forces for various Reynolds number.

The spacing between cylinders: $\mathrm{L}=4.2 \mathrm{D},(\mathrm{D}=40 \mathrm{~mm})$.

Measurement starts from the frontal point facing the mainstream flow and ends at the same point after a complete revolution. The average value is then calculated. The coefficient of pressure is defined as :

$$
C_{P}=\frac{p-p_{r e f}}{0.5 \times\left(\rho \cdot U_{g}^{2}\right)}
$$
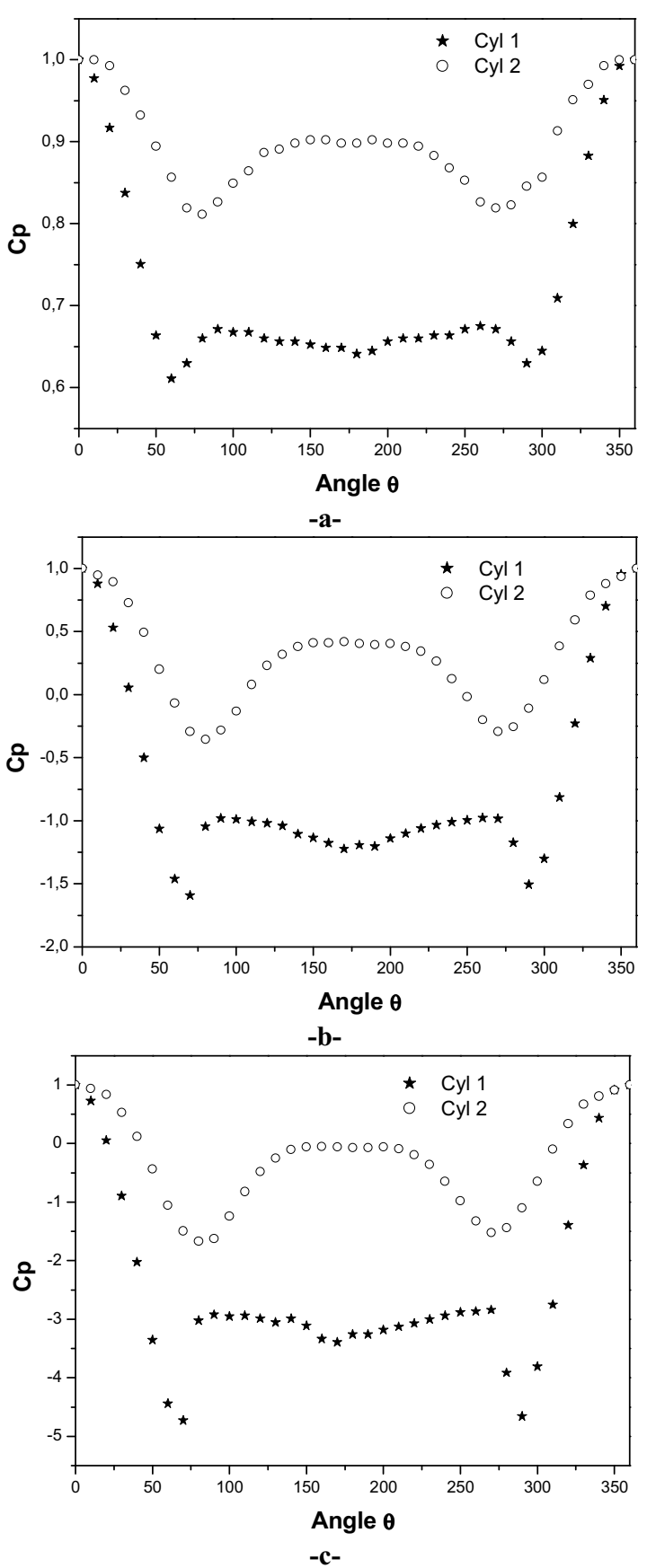

Fig. 3. Mean pressure coefficient distribution for the first case. (a) $\operatorname{Re}=2 \times 10^{4}$, (b) $\operatorname{Re}=5 \times 10^{4}$ and (c) $\operatorname{Re} 7 \times 10^{4}$.

For finite cylinders the mean pressure on the cylinder surface is also a direct result of free end interference. Park and Lee [14] reported that the mean 
pressure on the windward surface increases as we move to the fixed end. This is due to the fact that the vortices shed from two sides of the cylinder become dominant. On the leeward side however near the free end the mean pressure is greatly reduced.

For the cylinders with both ends fixed, it had been shown that the surface pressure distribution oscillates at the vortex shedding period and that pressure variations are consistent with periodic changes of the circulation around the cylinder body.

In Figure 3 and 4, mean pressure distributions around the two cylinders for the first and second case at the mid-span are presented.

For the both figures the pressure distribution on cylinders is almost a symmetry mapping resulting in a low lift force. In the pressure recovery region behind the cylinders the profile becomes flat. Similar behavior is also found by Lam and Fang [15]. It is observed that the upstream cylinder has a greater drag pressure compared to the downstream cylinder because the first cylinder is directly opposed to the flow inlet however the second one is in the wake of the latter. It is to be noticed that the pressure distributions for the tow cylinders seem to be similar to that of an isolated cylinder. From the figure it is clearly seen that the stagnation point is located at an angle of $0^{\circ}$ degree which correspond to the maximum pressure. The interaction between the cylinders appears to be negligible because of the distance $(\mathrm{L}=4.2 \mathrm{D})$.

The pressure coefficient distribution depends of the on variation of the Reynolds number. For the two studied cases the pressure coefficient increases in the absolute value by the rising of Reynolds number.

In Comparison with the two cases, it is clearly observed that the pressure drag for the second case (grooved-grooved) is reduced and this for the Reynolds number $\mathrm{Re}_{2}=5 \times 10^{4}$ and $\mathrm{Re}_{3}=7 \times 10^{4}$. This remark is in agreement with Ladjedel et al.[16] in their experimental investigation. They found that by using grooved cylinder the drag force is reduced for higher Reynolds (greater than $3.2 \times 10^{4}$ ).

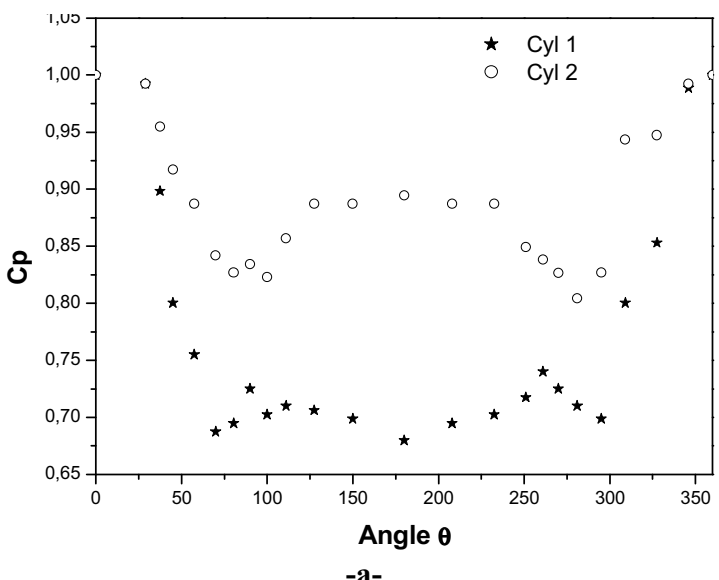

-a-

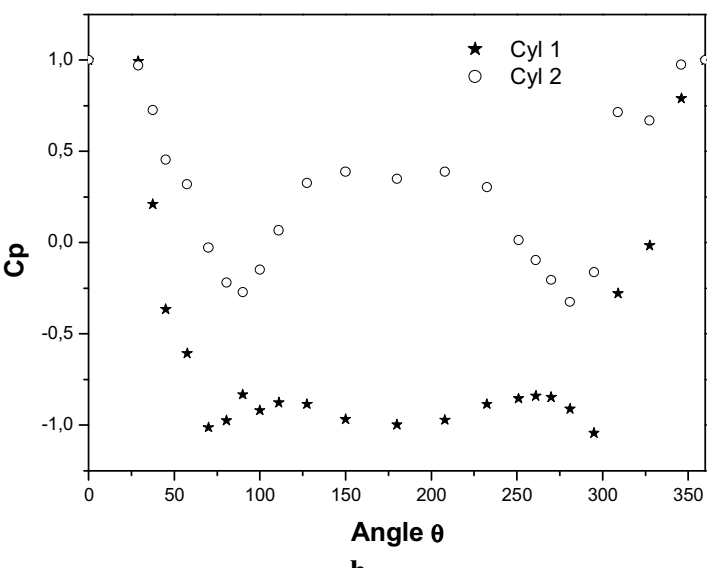

-b-

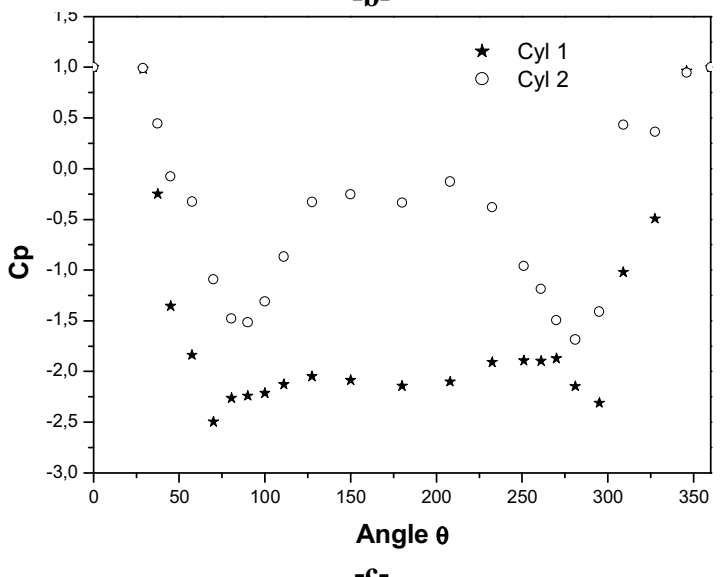

Fig.4. Mean pressure coefficient distribution for the second case. (a) $\operatorname{Re}=2 \times 10^{4}$, (b) $\operatorname{Re}=5 \times 10^{4}$ and (c) $\mathrm{Re}=7 \times 10^{4}$.

Table 1. The separation azimuthal position

\begin{tabular}{|c|c|c|c|c|c|c|c|}
\hline & \multicolumn{2}{|c|}{$\operatorname{Re}=2 \times 10^{4}$} & \multicolumn{2}{|c|}{$\operatorname{Re}=5 \times 10^{4}$} & \multicolumn{2}{|c|}{$\operatorname{Re}=7 \times 10^{4}$} \\
\hline & & $\theta$ & $\mathrm{C}_{\mathrm{P} \text { min }}$ & $\theta$ & $\mathrm{C}_{\mathrm{P} \min }$ & $\theta$ & $\mathrm{C}_{\mathbf{P \text { min }}}$ \\
\hline \multirow{4}{*}{ 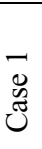 } & \multirow{2}{*}{$\begin{array}{c}\text { Cyl } \\
1\end{array}$} & $60^{\circ}$ & 0.611 & $70^{\circ}$ & -1.59 & $70^{\circ}$ & -4.73 \\
\hline & & $290^{\circ}$ & -0.63 & $290^{\circ}$ & -1.5 & $290^{\circ}$ & -4.66 \\
\hline & \multirow{2}{*}{$\begin{array}{c}\text { Cyl } \\
1\end{array}$} & $80^{\circ}$ & 0.812 & $80^{\circ}$ & -0.354 & $80^{\circ}$ & -1.67 \\
\hline & & $270^{\circ}$ & -0.82 & $270^{\circ}$ & -0.29 & $270^{\circ}$ & -1.523 \\
\hline \multirow{4}{*}{ 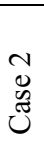 } & \multirow{2}{*}{$\begin{array}{c}\text { Cyl } \\
1\end{array}$} & $70^{\circ}$ & 0.68744 & $70^{\circ}$ & -1.013 & $70^{\circ}$ & -2.49 \\
\hline & & $280^{\circ}$ & 0.67991 & $295^{\circ}$ & -1.043 & $295^{\circ}$ & -2.31 \\
\hline & \multirow{2}{*}{$\begin{array}{c}\text { Cyl } \\
1\end{array}$} & $90^{\circ}$ & 0.823 & $90^{\circ}$ & 0.273 & $90^{\circ}$ & -1.52 \\
\hline & & $280^{\circ}$ & 0.8042 & $280^{\circ}$ & -0.33 & $280^{\circ}$ & -1.69 \\
\hline
\end{tabular}

Table 1 shows the minimum pressure coefficient values for different Reynolds numbers. It is clearly seen, there are two positions for $\mathrm{Cp}_{\text {min }}$ for all cases between $0^{\circ}$ and $360^{\circ}$. The angle corresponding to the pressure coefficient minimum value represents the separation of the reattached shear layer due to an increase in the pressure gradient. The separation creates a narrow wake. It is observed for the studied cases that the separation of the shear layer in the downstream cylinders is delayed compared to the upstream cylinder.

The drag coefficient is calculated using the following formula:

$$
C_{D}=\frac{2 \cdot D}{\rho \times U_{0}^{2} \times S}
$$

D: Drag force $(\mathrm{N})$,

S: Projected area of the cylinder $S=d \times L$ 
$\rho$ : Fluid density $\left(\mathrm{Kg} \mathrm{m}^{-3}\right)$.

$\mathrm{L}$ : cylinder Length (m).

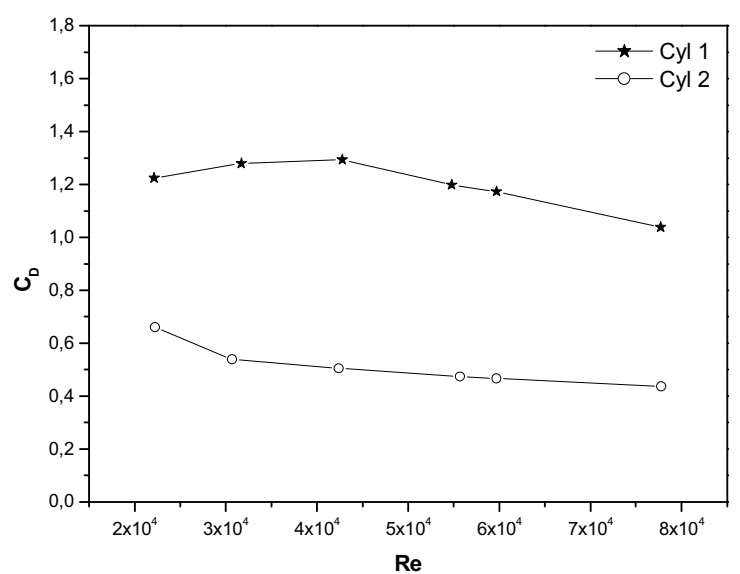

Figure 5. Drag coefficients distributions for the case one

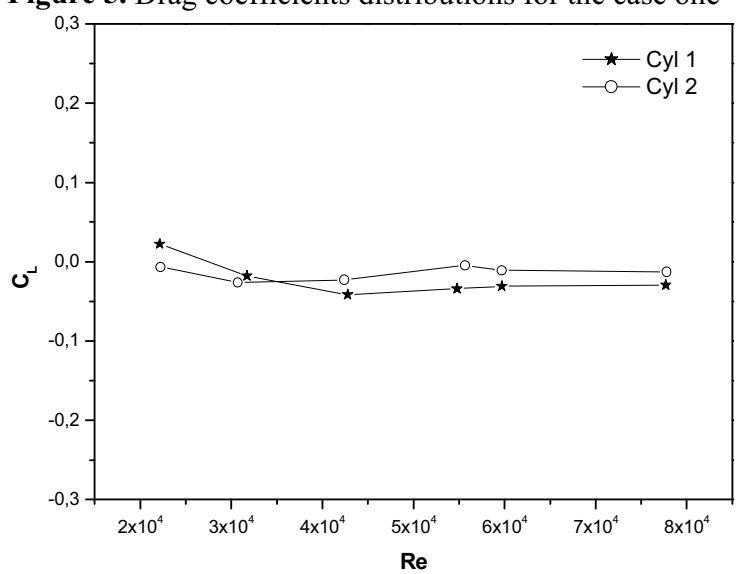

Fig.6. Lift coefficients distributions for the case one

Figure 5 and 6 show respectively the evolution of drag and lift coefficient for the case (smooth-smooth). It is seen that the drag force is higher in the upstream cylinder which is explained above. The lift distribution according to the Reynolds number seems to be negligible because of the distance between tubes and that confirms the symmetrical trend found in the pressure distribution.

\section{Conclusions}

The flow around two cylinders in tandem arrangement by using smooth and grooved cylinder was investigated. The results obtained seem to agree rather well.

Following this investigation and the subsequent conclusions are drawn:

- The pressure coefficient increases in the absolute value by the rising of Reynolds number.

- The upstream cylinder has a greater drag pressure compared to the downstream cylinder.

- The pressure distribution trend for the both cylinders seems to be the same as an isolated cylinder.

- The drag pressure is reduced for the high Reynolds number when the grooved cylinders are used.

\section{References}

1. M.M. Alam, M. Moriya, K. Takai, H. Sakamoto, J. Wind Eng. Ind. Aerodyn. 91 (1-2), 139 (2003)

2. M.M. Alam, H. Sakamoto, M. Moriya, J. Fluids Struct. 18 (3-4), 347 (2003)

3. T. Igarashi, Bull. JSME 24 (188), 323 (1981)

4. M. Arie, M. Kiya, M. Moriya, H. Mori, ASME J. Fluids Eng. 105, 161 (1983)

5. A. Okajima, Bulletin of JSME 22, 504 (1979)

6. M.M. Zdravkovich, Journal of Fluids and Structures 1, 239 (1987)

7. G. Xu, Y. Zhou. Experiments in Fluids 37, 248 (2004)

8. D. Brika, A. Laneville, Journal of Fluids and Structures 13, 579 (1999)

9. A. Laneville, D. Brika, Journal of Fluids and Structures 13, 967 (1999)

10. F.S. Hover, M.S. Triantafyllou, Journal of Fluids and Structures 15, 503 (2001)

11. G.R.S. Assi, J.R. Meneghini, J.A.P. Aranha, P.W. Bearman, E. Casaprima, Journal of Fluids and Structures 22, 819 (2006)

12. A. Bokaian, F. Geoola, Journal of Fluid Mechanics 146, 383 (1984)

13. O. Ladjedel, L. Adjlout, O. Imine, T. Yahiaoui, EPJ Web of Conferences 25 (01044), (2012)

14. C.W. Park, S.J. Lee, Journal of Wind Engg. and I. Aerodynamics 88. 231 (2000)

15. K. Lam, X. Fang, Journal of Fluids and Structures 9, 195 (1995)

16. O. Ladjedel, T.Yahiaoui, L.Adjlout and O.Imine, World Academy of Science, Engineering and Technology 53, 357 (2011) 